\title{
On the development and evolution of nonlinear ion acoustic wave packets
}

\author{
A. M. Hamza \\ Physics Dept., University of New Brunswick, POB 4400, Fredericton, E3B 5B-5A3, Canada \\ Received: 29 March 2005 - Revised: 6 June 2005 - Accepted: 8 June 2005 - Published: 15 September 2005
}

\begin{abstract}
A simple model of ion fluctuations (ion acoustic and ion cyclotron fluctuations for example) driven by an electron current which leads to intermittent fluctuations when the linear growth rate exceeds the wave packet dispersion rate is analized. The normalized fluctuation amplitude $e \phi_{0} / T$ can be much larger than the mass ratio $\left(m_{e} / m_{i}\right)$ level predicted by the conventional quasilinear theory or Manheimer's theory (see references in this document), and where $\phi_{0}$ represents the amplitude of the main peak of the ion fluctuations. Although the ion motion is linear, intermittency is produced by the strong nonlinear electron response, which causes the electron momentum input to the ion fluctuations to be spatially localized. We treat the 1-D case because it is especially simple from an intuitive and analytical point of view, but it is readily apparent and one can put forward the conjecture that the effect occurs in a three dimensional magnetized plasma. The 1-D analysis, as shown in this manuscript will clearly help identify the subtle difference between turbulence as conventionally understood and intermittency as it occurs in space and laboratory plasmas.
\end{abstract}

Keywords. Meteorology and atmospheric dynamics (Turbulence) - Ionosphere (Wave-particles interactions) - Space plasma physics (Waves and instabilities)

\section{Introduction}

\subsection{Historical background}

Isolated coherent fluctuations have been observed in both physical and simulation plasma. When such fluctuations are formed randomly in the presence of lower level background turbulence, the turbulence is called intermittent. A quantitative measure of intermittency is the Kurtosis, which is a measure of the flatness of the probability distribution function (see for example Frisch, 1995). There are many examples of such intermittent fluctuations, but here we shall be mainly

Correspondence to: A. M. Hamza

(ahamza@unb.ca) interested in a very simple example, namely 1-D ion fluctuations driven by an electron current (in the ion acoustic and ion cyclotron regimes), illustrating the development of large and coherent ion fluctuations in a current driven plasma. Our preliminary studies indicate that the 1-D effects dealt with here persist when extended to the case of a 3-D plasma in a magnetic field (see for example Hamza, 1988, 1993). Although the 1-D model is limited in direct application to physical problems, this deficiency is more than compensated for by the relative simplicity of the analytic treatment and the more definitive and unambiguous nature of the relevant computer simulations. It is also illuminating that even in this simplest of cases, the conventional theoretical understanding is wrong.

Sato and Okuda $(1979,1981)$ were apparently the first to observe such fluctuations in numerical simulations. Barnes et al. (1985) obtained similar results but provide much more detailed phase space information. Berman et al. (1986) extended the work to include additional diagnostics and to show that such fluctuations could occur in linearly stable plasma. These simulations were motivated, in part, by satellite observations (see for example Koeskinen et al. (1988) and Mozer et al. (1982)) of isolated large amplitude fluctuations in auroral plasma thought to be driven by an electron current (see for example Mozer et al. (1982)). More recently a number of satellite observations from Freja and FAST have clearly identified intermittent, spatially localized ion fluctuations (see for example Potelette et al. (2004) and references therein).

In particular we are interested in the early stages of the simulations in which the ion acoustic wave is linearly unstable. In these simulations, isolated wave-like fluctuations occur at an early stage when the amplitude is low and the ion motion is clearly linear. These fluctuations ultimately grow to very large amplitude, trap ions, and turn into BGK holes (see Bernstein et al. (1957)). This later phase of the simulation seems to be well understood (see for example Dupree (1986) and references therein). The question we address here is the nature of early time isolated low amplitude fluctuations. 


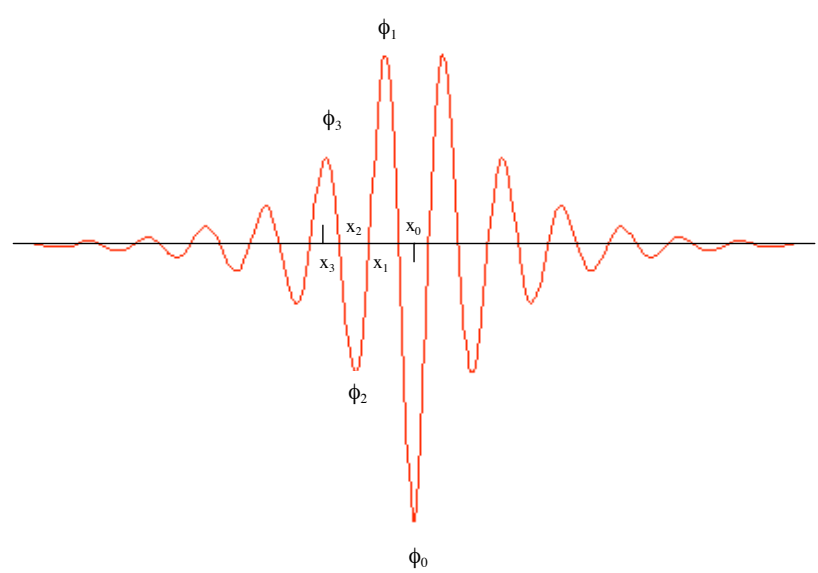

Fig. 1. A symmetric wave Packet illustration with maxima and minima.

There are essentially two questions to be addressed. First, how can the fluctuations grow to the large amplitudes, of order $e\left|\phi_{0}\right| / T \approx 1$ (noting that large amplitudes can exceed the value of one), observed in the simulations when the conventional analysis predicts amplitudes of order $e\left|\phi_{0}\right| / T \approx m_{e} / m_{i}$ or less? $\left(\phi_{0}\right.$, which is negative in the case of interest, is the value of the potential $\phi(x)$ at its maximum amplitude (see Fig. 1), $T$ is the electron temperature, and the other symbols have their usual meaning.) And secondly, what is the physical nature of the localized ion fluctuation?

\subsubsection{Conventional answers}

The first question involves electron free energy (or momentum) and the relaxation of the electron distribution function by the potential fluctuations (see for example reference Dupree, 1986). Historically there have been two treatments of this question depending on the ratio of the fluctuation autocorrelation rate $\gamma_{c}$ to the electron reflection or trapping rate $\gamma_{e}$. When $\gamma_{c} \gg \gamma_{e}$, quasi linear theory applies and predicts that the ultimate fluctuation level (in a closed system) is very small, of order $e\left|\phi_{0}\right| / T \approx m_{e} / m_{i}$ or less. Manheimer (1971) has analyzed the other limit, $\gamma_{c} \ll \gamma_{e}$. His analysis predicts that the ultimate value of the potential is of the same order as the previous case.

That these two seemingly disparate approaches should both predict very small final fluctuation amplitude is not surprising. In both cases, the growth of the ion fluctuation is determined by the momentum lost by the relaxation ("plateauing") of the electron distribution function over a small fraction of its width in velocity space, i.e. of order $\left(m_{e} / m_{i}\right)^{1 / 2}$ or less. Quasilinear theory and the Manheimer theory treat a problem in which ion fluctuations are closely packed and fill the physical space. When the momentum lost by the electrons is uniformly allocated to the closely packet ion fluctuations to determine the amount absorbed by each fluctuation and therefore the amplitude of each fluctuation, one obtains the amplitude estimates given above.

\subsubsection{The intermittency argument}

Consider, however, the case in which the ion fluctuations are not closely packed. It is obvious that if the same amount of lost electron momentum is allocated to a smaller number of isolated ion fluctuations, the amplitude of each fluctuation must necessarily be larger. Furthermore there is an additional effect that leads to an even greater enhancement of fluctuation level. The momentum imparted to the ions comes from the relaxation of the electron distribution function over a velocity interval, $\Delta v_{e}$, equal to the trapping width $\left(e\left|\phi_{0}\right| / m_{e}\right)^{1 / 2}$. In the isolated fluctuation case the potential is no longer limited to a small value and as it increases so does the electron trapping width and therefore the electron free momentum available to drive the ion fluctuations. The amplitude pulls itself up by its bootstraps.

Following the conventional approach (see for example Drummond, 1965; Kadomtsev, 1965), one can make an approximate estimate of the final amplitude for isolated fluctuations by generalizing Manheimer's argument. We consider a plasma which initially contains an electron current and an array of negative potential pulses a distance $d$ apart (see Fig. 1). In the rest frame of the potential pulses, an electron whose speed is less than the trapping width $\Delta v_{e} \approx\left(e\left|\phi_{0}\right| / m_{e}\right)^{1 / 2}$ will be reflected by the potential pulses. Electrons moving in both directions will be reflected, but because there is a current, there will be a net momentum loss by the electrons and therefore a net momentum gain by the ion pulses and consequently the ion fluctuations will grow. As the pulse potential grows so does the maximum electron speed, $\Delta v_{e}$, that can be reflected. If the potential is growing exponentially at the rate $\gamma$, then during each successive time interval of length $\gamma^{-1}$ the potential increases by a factor of $e$ times its previous value and a whole new additional group of electrons can be reflected. This process will continue until the bulk of the electrons being reflected by a pulse have been previously reflected by another pulse. This would require that in one time interval, $\gamma^{-1}$, a reflected electron traveled a distance $d$. Thus the fluctuations will cease growing when $\gamma^{-1} \Delta v_{e} \approx d$. If the fluctuations are not isolated, but consist of an infinite wave train, then $d$ is set equal to the reciprocal wavenumber $k$ and one obtains Manheimer's criterion. On the other hand since $\phi_{0} \propto \Delta v_{e}^{2}$, making $d>k^{-1}$ enhances the potential fluctuation over the Manheimer value by the factor $(k d)^{2}$. By making $d$ sufficiently large one may obtain fluctuations with $e\left|\phi_{0}\right| / T \approx 1$, or larger, as convincingly demonstrated by the simulations.

The preceding argument shows how isolated fluctuations can grow to large amplitude, but it does not explain why a physical system produces isolated (as opposed to closely spaced) fluctuations in the first place. This feature can also be understood from the preceding arguments. A fluctuation grows until the electrons it reflects have been reflected by another fluctuation. Thus growing fluctuations compete with each other for electron momentum. But the larger fluctuations have an advantage in this contest and can grow at the expense of the smaller ones. The larger fluctuations can reflect, 
and are therefore driven by, electrons whose speeds are too great for them to have been reflected by smaller fluctuations, so the growth of the big fluctuations is not inhibited by the smaller ones. On the other hand, the converse is not true, the larger fluctuation do shadow the smaller ones and deprive them of momentum. Therefore as time evolves, only progressively larger fluctuations survive, and these, of necessity, must be progressively further apart since otherwise they would have shadowed each other at an earlier epoch in their growth and could not have grown to large amplitude. It is a case of "survival of the fittest".

\subsubsection{The ion acoustic wave packet case}

The point of the preceding discussion is that if isolated fluctuations exist, one can explain their apparent anomalously large amplitude. The open issue, to be addressed here, is to explain the nature of the observed ion fluctuations when they still have small amplitude, are localized, and have a negative potential peak. Not only is the negative potential peak observed in the auroral and simulation plasma, but it is theoretically necessary in order to reflect electrons and thereby obtain a momentum input to the fluctuations to make them grow. There are several obvious candidates for the observed fluctuations. As mentioned earlier, BGK holes qualify, but we are interested in amplitudes which are too small to trap ions. Another candidate, frequently proposed in the literature, are Kd.V solitons. However these fail on several counts. First the compressive Kd.V soliton has a positive potential peak, not the required negative peak. Also the Kd.V soliton propagates with a speed that is amplitude dependent contrary to the results of the simulations. Finally the localization of the compressive $\mathrm{Kd}$.V soliton is achieved by balancing the dispersion against ion nonlinearities. However, the simulations show localized fluctuations at small amplitudes where the ions are linear.

Another possibility is a linear wave packet. This candidate would also appear to fail since dispersion will cause such a packet to decay in amplitude and spread out in space at a rate $\gamma_{d}$ which is rapid compared to the observed lifetimes in the simulations. Nevertheless it is possible to make the linear wave packet work. The principal result of this paper is that when the linear growth rate exceeds the dispersion rate, an electron nonlinearity (as contrasted to an ion nonlinearity) will counteract the effects of dispersion and keep the fluctuation localized.

The physical basis of this effect is quite simple, although unexpected. In Sect. 2 we consider electron reflection and trapping in a growing wave packet consisting of a central peak with progressively smaller "foothills" (lower amplitude wave packet peaks, see Fig. 1) on either side of the main peak. We show that, although electron reflection occurs at all peaks including the main peak, the net momentum input to the ions occurs only around the main peak. Since, for linear ions, fluctuation amplitude is proportional to momentum content, this means that the electrons drive only the main peak of the wave packet. There is no momentum input by the electrons to the "foothills" since the input by reflected electrons is canceled by electrons trapped between the "foothills". As the electrons feed momentum into the main peak, dispersion will feed momentum into the foothills causing their amplitude to grow. However, if electron momentum is fed into the main peak faster than it is lost by dispersion, the main peak will grow.

\subsubsection{Linear theory of ion acoustic instability}

The linear theory of ion acoustic waves is elementary and well known so we shall only quote the results here. For a wave of frequency $\omega$ and wavenumber $k$, the real part of the appropriate dielectric function for $v_{i} \ll \omega / k \ll v_{e}$ is

$\epsilon_{R}(k, \omega)=1+\left(k^{2} \lambda_{D}^{2}\right)^{-1}-\omega_{p i}^{2} / \omega^{2}$

where $\epsilon_{R}, \lambda_{D}$, and $\omega_{p i}$ are the real part of the dielectric function, the electron Debye length and the ion plasma frequency respectively. Setting $\epsilon_{R}=0$ produces the dispersion relation

$\omega_{k}^{2}=k^{2} c_{s}^{2}\left(1+k^{2} \lambda_{D}^{2}\right)^{-1}$

where $c_{S}=\omega_{p i} \lambda_{D}=\left(T / m_{i}\right)^{1 / 2}$ is the sound speed. When the imaginary part of $\epsilon$ is small, the linear growth rate of ion acoustic waves $\gamma_{\ell}$ is given by

$\gamma_{\ell}=(\pi / 2) k c_{s} v_{e}^{2} f_{0 e}^{\prime}(u)$

where $v_{e}=\lambda_{D} \omega_{p e}$ is the electron thermal speed, $f_{0 e}^{\prime}(u)=(\partial / \partial u) f_{0 e}(u)$, and $f_{0 e}(u)$ is the unperturbed electron distribution function evaluated at the phase speed of the wave packet $u=\omega / k$.

The broadening of a linear wave packet due to dispersion is treated in reference (Jackson, 2000), but the essential results are easy to derive by approximate arguments. We define the dispersion rate $\gamma_{d}$ as one-half the rate of increase of the logarithm of the width of the wave packet. For a wave packet containing a wave number spread of $\Delta k$, the spread in group velocity is $\Delta k\left(\partial^{2} \omega_{k} / \partial k^{2}\right)$. If the spatial width of the wave packet is $\pi / \Delta k$ the dispersion rate will be $\gamma_{d} \approx k^{2}\left(\partial^{2} \omega_{k} / \partial k^{2}\right) / 2 \pi$. Using Eq. (2), we find

$\gamma_{d} \approx \frac{3}{2}\left(k \lambda_{D}\right)^{3} \omega_{p i} / \pi$.

1.1.5 On the evolution of a linear ion acoustic wave packet

In the absence of any driving mechanism, the wave packet envelope will broaden at the rate $2 \gamma_{d}$. The total energy and momentum of the wave packet is proportional to the spatial integral of the potential squared over the length of the envelope. To conserve energy and momentum the amplitude of the envelope will have to decrease at the rate $\gamma_{d}$. This means that dispersion will cause momentum to be lost (and spread) from the main peak at the rate $2 \gamma_{d}$.

We show in Sect. 3) that electrons whose distribution function in the absence of a wave packed is $f_{0 e}(v)$ will transfer momentum to a wave packet whose peak potential is $\phi_{0}$ at the rate

$\dot{P}=\omega_{p e^{\pi}}^{2} f_{0 e}^{\prime}(u) \phi_{0}^{2}$ 
where $u$ is the phase velocity of the wave packet. The rate of total momentum loss $\dot{P}$ depends only on the maximum potential $\phi_{0}$, not on the shape or length of the wave packet. However, as we have explained, the lost electron momentum is all deposited in the spatial region containing the main peak of the wave packet. The foothills get no net momentum directly from the electrons, they get it only through dispersion.

If all the momentum lost from the electrons goes into the main peak, the rate of change of fluctuation momentum, $\dot{P}$, can be related to the rate of change of potential using the standard relation

$\dot{P}=\frac{k^{2}}{4 \pi} \frac{\partial \epsilon(k, k u)}{\partial u} \frac{d}{d t} \int d x \phi^{2}$

where the integral is over the width of the main peak. Using $k^{2} \partial \epsilon / \partial u=2\left(c_{s} \lambda_{D}^{2}\right)^{-1}$ and $\int d x \phi^{2} \approx(\pi / 2 k) \phi_{0}^{2}$ this may be written

$\dot{P}=\left(2 \pi c_{s} \lambda_{D}^{2}\right)^{-1} \frac{\partial}{\partial t} \int d x \phi(x)^{2} \approx\left(4 k c_{s} \lambda_{D}^{2}\right)^{-1} \frac{d}{d t} \phi_{0}^{2}$.

Equating (5) and (7) and solving for $\gamma=\phi_{0}^{-1}\left(d \phi_{0} / d t\right)$ we obtain $\gamma=\gamma_{\ell}$.

As explained earlier, growth due to electron reflection is not the only thing affecting the main peak. It is also losing momentum and amplitude due to dispersion at the rate $\gamma_{d}$. Therefore the amplitude of the main peak $\phi_{0}$ will grow at the approximate rate

$\gamma \approx \gamma_{\ell}-\gamma_{d}$.

For the amplitude of the main peak to have a positive growth rate $(\gamma>0)$ it is necessary that

$\gamma_{\ell} / \gamma_{d}>1$.

The rates $\gamma_{\ell}$ and $\gamma_{d}$ both depend on the average wavenumber $k$. The ratio $\gamma_{\ell} / \gamma_{d}$ is proportional to $k^{-2}$. Clearly $\gamma_{\ell} / \gamma_{d}$ can be made arbitrarily large by making $k$ small enough. On the other hand the growth rate $\gamma$ given by Eq. (9) decreases for small $k$. In fact it is easy to show that $\gamma$ has a maximum at $\left(k \lambda_{D}\right)^{2}=\left(\pi^{2} / 6\right) v_{e}^{2} f_{0 e}^{\prime}, \gamma_{\ell} / \gamma_{d}=3, \gamma / \gamma_{d}=2$, and $\gamma=(2 / 3) \gamma_{\ell}$. These values are in reasonable agreement with the simulations (for $v_{e}^{2} f_{0 e}^{\prime} \approx 0.5, \pi / k \approx 15 \lambda_{D}$ ). Fluctuations with this value of $k$ would dominate since they would grow fastest and shadow the slower growing fluctuations.

\section{The wave packet equation}

The basic equations that describe the 1-D system under consideration are the Vlasov and Poisson equations:

$$
\begin{aligned}
\frac{\partial f_{e}(x, v, t)}{\partial t}+v \frac{\partial f_{e}(x, v, t)}{\partial x} & +\frac{e}{m_{e}} \frac{\partial \phi(x, t)}{\partial x} \frac{\partial f_{e}(x, v, t)}{\partial v}=0
\end{aligned}
$$

and

$$
\frac{\partial^{2} \phi(x, t)}{\partial x^{2}}=4 \pi e \int_{-\infty}^{+\infty} d v f_{e}(x, v, t)-4 \pi q_{i} n_{i}
$$

which describe the evolution of the electron distribution function $f_{e}(x, v, t)$ and the electrostatic potential $\phi(x, t)$.

In Poisson's equation (Eq. (11)) we assume that electrons whose energies $E=m_{e} v^{2} / 2-e \phi(x)$ are greater than $-e \phi_{0}$ can be described by the linear theory. This portion of the electron charge density and the entire ion charge density can then be included in the real part of the dielectric function $\epsilon_{R}(k, \omega)$. The trapped and reflected electrons with energies such that $E<-e \phi_{0}$ contribute nonlinear terms that can be separated from the linear ones. Following the standard procedure (see for example Denavit and Sudan, 1972; Karpman, 1979; Taniuti, 1974), the Fourier transform in space and time of the linearized Vlasov equation and Poisson's equation become

$k^{2} \epsilon_{R}(k, \omega) \phi(k, \omega)=-4 \pi e \tilde{n}_{e}(k, \omega)$

where $\tilde{n}_{e}(k, \omega)$ is the Fourier transform of

$\tilde{n}_{e}(x, t)=\int_{E<-e \phi_{0}} d v\left[f_{e}(x, v, t)-f_{0 e}(v)\right)$

and the integration is limited to the energy range $E<-e \phi_{0}$. This means that the charge density on the right hand side includes all electrons not included in the linear portion included in $\epsilon_{R}(k, \omega)$. Also included in the integral is the charge neutralizing portion of the ions equal to $-f_{0 e}(v)$. Expanding the real part of the dielectric function in Eq. (12) around the ion acoustic frequency $\omega_{k}$ given by Eq. (2) leads to:

$\left(\omega-\omega_{k}\right) \phi(k, \omega)=-\frac{4 \pi e \tilde{n}_{e}(k, \omega)}{k^{2} \partial \epsilon_{R}\left(k, \omega_{k}\right) / \partial \omega_{k}}$.

The frequency may be approximated by expanding Eq. (2), $\omega_{k}=k c_{s}-k^{3} c_{s} \lambda_{D}^{2} / 2+\cdots$. Without loss of generality we have retained only the positive frequency root of $\epsilon_{R}(k, \omega)=0$. Since we assume that $\tilde{n}_{e}(k, \omega)$ is already of the same order as $\omega-\omega_{k}$, (i.e. of order $\gamma \approx \gamma_{d} \approx \gamma_{e}$, where $\gamma$ represents the growth rate of the main peak $\phi_{0}, \gamma_{d}$, the dispersion rate, and $\gamma_{e}$ the electron reflection or bounce rate) we may compute $\partial \epsilon_{R}(k, \omega) / \partial \omega$ to lowest order in $k^{2} \lambda_{D}^{2}$ to obtain $\partial \epsilon_{R}\left(k, \omega_{k}\right) / \partial \omega_{k}=2\left(k c_{s} \lambda_{D}^{2}\right)^{-1}\left(1+k^{2} \lambda_{D}^{2}\right)^{3 / 2} \approx 2\left(k c_{s} \lambda_{D}^{2}\right)^{-1}$.

With these approximations and multiplying by $-i$ Eq. (14) becomes:

$$
\begin{gathered}
-i \omega \phi(k, \omega)+i k c_{s} \phi(k, \omega)+\frac{c_{s}}{2} \lambda_{D}^{2}(i k)^{3} \phi(k, \omega) \\
=i k c_{s}\left(\lambda_{D}^{2} / 2\right) 4 \pi e \tilde{n}_{e}(k, \omega) .
\end{gathered}
$$

An inverse Fourier transformation of this equation produces the equation governing the evolution of the electrostatic potential $\phi(x, t)$ :

$$
\begin{aligned}
& \frac{\partial \phi(x, t)}{\partial t}+c_{s} \frac{\partial \phi(x, t)}{\partial x}+\frac{1}{2} c_{s} \lambda_{D}^{2} \frac{\partial^{3} \phi(x, t)}{\partial x^{3}} \\
& =2 \pi c_{s} \lambda_{D}^{2} \frac{\partial}{\partial x} e \tilde{n}_{e}(x, t) .
\end{aligned}
$$

The left hand side of this equation is basically the linear Korteweg de Vries equation which describes the linear evolution of ion acoustic wave packets. The term on the right hand side 
of the equation accounts for the nonlinear response of the electrons, namely the response of the trapped, and reflected electrons.

Unfortunately $\tilde{n}_{e}(x, t)$ turns out to be very complicated in the nonlinear case so that Eq. (16) is virtually impossible to solve analytically. However some insight concerning the growth of $\phi(x)$ can be gained by multiplying Eq. (16) by $\left(2 \pi c_{s} \lambda_{D}^{2}\right)^{-1} \phi(x)$ and integrating over $x$ from $x$ to $+\infty$. This gives an equation for the rate of change of wave packet momentum (and therefore amplitude) in the region between $x$ and $\infty$.

$\left(2 \pi c_{s} \lambda_{D}^{2}\right)^{(-1)} \frac{\partial}{\partial t} \int_{x}^{+\infty} d x \phi(x)^{2}-J(x)=S_{+}(x)$

where

$$
\begin{aligned}
J(x) & =\left(4 \pi \lambda_{D}^{2}\right)^{-1} \phi(x)^{2} \\
+ & \frac{1}{4 \pi}\left[\phi(x) \frac{\partial^{2}}{\partial x^{2}} \phi(x)-(1 / 2)\left(\frac{\partial \phi(x)}{\partial x}\right)^{2}\right] \\
S_{+}(x) & =\dot{P}_{+}(x)-e \tilde{n}_{e}(x) \phi(x)
\end{aligned}
$$

and

$$
\dot{P}_{+}(x)=-e \int_{x}^{+\infty} d x \tilde{n}_{e}(x) \frac{\partial}{\partial x} \phi(x) .
$$

The quantity $J$ is a momentum current. The first term in $J$ is due to the wave phase velocity, the second term arises from dispersion and is always negative if the potential is always concave towards the $\mathrm{x}$ axis. We have assumed that $\phi(\infty)=0$.

The quantity $S_{+}(x)$ is the rate of momentum input to the wave packet by the trapped and reflected electrons in the region $x_{0}<x<\infty$ (i.e. located to the right of the main peak of the wave packet). The quantity $-e \tilde{n}_{e}(x) \phi(x)$ is a momentum current due to the trapped and reflected electrons. In the next Section we will show that, when the nonlinear response of the electrons is taken into account, $S_{+}(x)$ vanishes for $x=x_{+}\left(S_{+}\left(x_{+}\right)=0\right)$ where $x_{+}$lies between $x_{0}$ and the value of $x$ at the first zero of the potential $\phi(x)$ to the right of $x_{0}$ (see Fig. 2). If $S_{+}(x)$ vanishes at $x_{+}$, then the electrons make no contribution to the growth of $\phi(x)$, as measured by $\int_{x}^{\infty} d x^{\prime} \phi\left(x^{\prime}\right)^{2}$, in the region $x_{+}<x<\infty$. Thus the electrons cause the potential to grow only in the region of the central peak. Of course dispersion as described by the second term of $J$ in Eq. (18) will cause momentum to flow out of this region.

It is enlightening to contrast the nonlinear case with the linear theory. It is easy to show that in the linear theory that if $\phi(x)=\phi_{0} \cos (k x)$ then the component of the density in phase with $\partial \phi / \partial x$ is $\tilde{n}_{e}=\gamma_{\ell} \phi_{0} \sin (k x)$. For this case it is clear that the electron source term $S_{+}(x)$ will not have zeros but in fact will be proportional to $\int_{x}^{\infty} d x \sin (x)^{2}$, expressing uniform growth along the entire (infinite) spatial extent of the wave packet.

An equation analogous to Eq. (17) can be derived for the region $x<x_{0}$. We obtain

$$
\left(2 \pi c_{s} \lambda_{D}^{2}\right)^{(-1)} \frac{\partial}{\partial t} \int_{-\infty}^{x} d x \phi(x)^{2}+J(x)=S_{-}(x)
$$

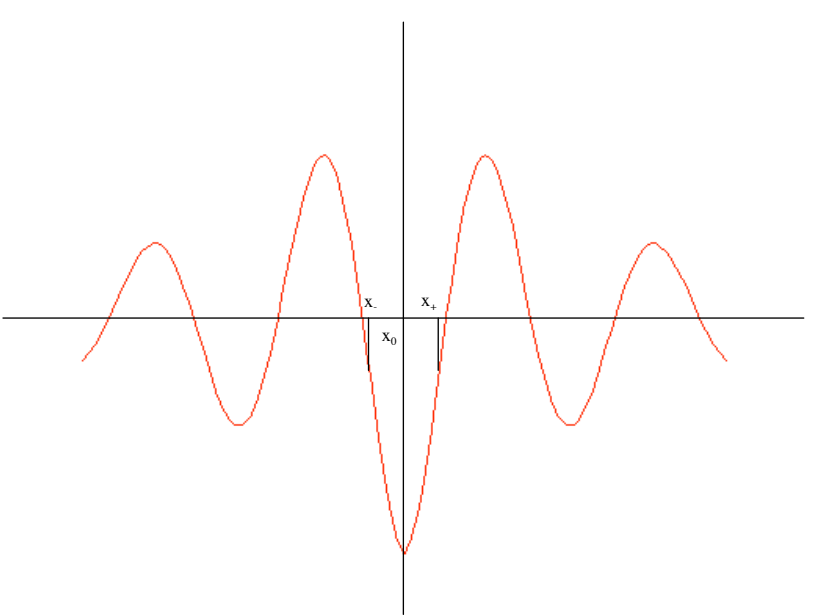

Fig. 2. The positions $x+$ and $x$ - related to the zeroes of momentum input.

where

$S_{-}(x)=\dot{P}_{-}(x)+e \tilde{n}_{e}(x) \phi(x)$

and

$\dot{P}_{-}(x)=-e \int_{-\infty}^{x} d x \tilde{n}_{e}(x) \frac{\partial}{\partial x} \phi(x)$.

In analogy to $S_{+}(x), S_{-}(x)$ has a zero at $x=x_{-}<x_{0}$ $\left(S_{-}\left(x_{-}\right)=0\right)$ where $x_{-}$is the first zero of the potential $\phi(x)$ on the left hand side of $x_{0}$.

Using $\quad \int_{x_{-}}^{x_{+}}=\int_{-\infty}^{x_{0}}+\int_{x_{0}}^{\infty}-\int_{-\infty}^{x_{-}}-\int_{x_{+}}^{\infty} \quad$ and $S_{+}\left(x_{+}\right)=S_{-}\left(x_{-}\right)=0$ we can obtain the rate of change of $\phi(x)^{2}$ averaged over the region $x_{-}<x<x_{+}$

$$
\begin{aligned}
& \left.\frac{\partial}{\partial t}\left(2 \pi c_{s} \lambda_{D}^{2}\right)^{-1} \int_{x_{-}}^{x_{+}} d x \phi(x)^{2}+J\left(x_{-}\right)-J_{(} x_{+}\right) \\
& \quad=\dot{P}_{-}\left(x_{0}\right)+\dot{P}_{+}\left(x_{0}\right) .
\end{aligned}
$$

Since $S_{-}\left(x_{0}\right)+S_{+}\left(x_{0}\right)=\dot{P}_{-}\left(x_{0}\right)+\dot{P}_{+}\left(x_{0}\right)$ is the total momentum lost by the electrons to the wave, this equation shows that all of the lost electron momentum is deposited in the spatial region of the main wave packet peak $x_{-}<x<x_{+}$.

\section{Spatial dependence of electron momentum input to wave packet}

Our objective in this section is to show that $S_{+}(x)$ and $S_{-}(x)$ have zeros at $x_{+}$and $x_{-}$respectively. Although the electron response to the wave potential is very nonlinear, the calculation is tractable when the growth rate $\gamma$ and the dispersion rate $\gamma_{d}$ are comparable and both are much less than the electron reflection or bounce rate $\gamma_{e}$

$\gamma \approx \gamma_{d} \ll \gamma_{e}$

where

$\gamma_{e}=k\left(\frac{-2 e \phi_{0}}{m_{e}}\right)^{1 / 2}=k \lambda_{D} \omega_{p e}\left(\frac{-e \phi_{0}}{T}\right)^{1 / 2}$. 
Using Eq. (4) the condition $\gamma_{e} \gg \gamma_{d}$ can be expressed as a restriction on the amplitude of the wave packet, i.e.,

$\frac{-e \phi_{0}}{T} \gg \pi^{-2}\left(k \lambda_{D}\right)^{4}\left(\frac{m_{e}}{m_{i}}\right)$.

Therefore with $k^{-1} \simeq 10 \lambda_{D}$ (the value observed in the simulations), $\gamma_{e} \gg \gamma_{d}$ requires

$$
\frac{-e \phi_{0}}{T} \gg 10^{-5}\left(\frac{m_{e}}{m_{i}}\right)
$$

which is an exceedingly small amplitude level. The significance of $\gamma_{e} \gg \gamma_{d}$ is that in this case the electron energy

$$
E=m_{e} v^{2} / 2-e \phi(x)
$$

is approximately constant in the rest frame of the wave packet making it easy to solve for the electron dynamics in terms of trapped, reflected, and passing particles.

To evaluate $S_{+}(x)$ we introduce the perturbed electron distribution function

$\tilde{f}_{e}(x, v)=f_{e}(x, v)-f_{0 e}(v)$

Next we go to the rest frame of the wave packet $(u=0)$ and change the velocity integration into an energy integral

$\tilde{n}_{e}(x)=2 \int_{-e \phi\left(x^{\prime}\right)}^{-e \phi_{0}} \frac{d E \tilde{f}_{e}(E, t)}{\left[2 m_{e}\left(E+e \phi\left(x^{\prime}\right)\right)\right]^{1 / 2}}$.

When a distribution function contains the argument $E$, it is to be understood that the velocity argument $v$ has been replaced with $v(E)=\left[\left(2 / m_{e}\right)(E+e \phi(x))\right]^{1 / 2}$, for example $\tilde{f}_{e}(E)=\tilde{f}_{e}(v(E))$. The quantiy $\dot{P}_{+}(x)$ given by Eq. (20) can be written

$$
\dot{P}_{+}(x)=-2 e \int_{x}^{+\infty} d x^{\prime} \frac{\partial \phi\left(x^{\prime}\right)}{\partial x^{\prime}} \int_{-e \phi\left(x^{\prime}\right)}^{-e \phi_{0}} \frac{d E \tilde{f}_{e}(E, t)}{\left[2 m_{e}\left(E+e \phi\left(x^{\prime}\right)\right)\right]^{1 / 2}} .
$$

One must bear in mind that $\tilde{f}_{e}(E, t)$ in Eq. (32) is more complex than it appears since in general it is a different function of $E$ in different spatial regions. In other words $\tilde{f}_{e}(E, t)$ has an implicit $x$ dependence. However it is possible to break the spatial integration in multiple regions in each of which $\tilde{f}_{e}(E, t)$ is not $x$ dependent. In other words, we will use the fact that phase space density is conserved along particle orbits.

For the purpose of calculating momentum input to the wave packet by the electrons, we shall assume the packet consists of a central potential energy peak, $-e \phi_{0}$ located at $x=x_{0}$ with a series of progressively smaller potential energy peaks ("foothills") on either side. Because of the negative electron charge, we focus on the potential energy instead of potential. Progressively smaller means that the peak amplitudes are monotonically decreasing functions of $\left|x-x_{0}\right|$. The foothill peak immediately to the right of the main peak is located at $x=x_{1}$ and has the potential value $\phi\left(x_{1}\right)=\phi_{1}$. Successive peaks occur at $x_{2}, x_{3}, \cdots$ and have potential values $\phi_{2}$, $\phi_{3}, \cdots$ In general, the distribution function can be a different function of $E$ in the intervals $\left(x_{0}<x<x_{1}\right),\left(x_{1}<x<x_{2}\right)$, $\left(x_{2}<x<x_{3}\right)$, etc. We shall refer to these as intervals $1,2,3$, etc. respectively. In each spatial interval there are two energy regions for $E<-e \phi_{0}$. One energy region consists of those electrons which come from $x=+\infty$ and are reflected back to $x=+\infty$. We refer to these electrons as "reflecting" electrons. For reflecting electrons, $\tilde{f}_{e}(E)$ has the same value it has at $x=+\infty$ namely $\tilde{f}_{0 e}^{+}(E)$. The other energy region consists of trapped electrons whose distribution can in principle be described arbitrarily, but in the present problem can be related to $\tilde{f}_{0 e}^{+}(E)$ since these electrons originally came from $x=+\infty$ and were, at an earlier time, trapped by the growing potential. For example, in spatial region 1 , reflecting electrons are those for which $-e \phi_{1}<E<-e \phi_{0}$ and the trapped electrons have $E<-e \phi_{1}$.

Although $\tilde{f}_{e}(E)$ is a different function of $E$ in each of the spatial intervals, within each spatial interval it is not a function of $x$. Therefore the $x^{\prime}$ integral range $x<x^{\prime}<+\infty$ in Eq. (32) may be broken into a sum of integrals over intervals in which $\tilde{f}_{e}(E)$ is independent of $x$. Denoting the $i$ th interval by $i$, and its upper and lower $x$ boundary by $u_{i}$ and $l_{i}$ (i.e. in the $i$ th interval $l_{i}<x<u_{i}$ ) we can write the integral in Eq. (32) as

$$
\begin{aligned}
& \dot{P}_{+}(x)= \\
& -\sum_{i} \int_{l_{i}}^{u_{i}} d x^{\prime} \frac{\partial}{\partial x^{\prime}} \int_{-e \phi\left(x^{\prime}\right)}^{-e \phi_{0}} d E\left[\frac{2}{m_{e}}\left(E+e \phi\left(x^{\prime}\right)\right)\right]^{1 / 2} \tilde{f}_{e}^{i}(E, t) \\
& =\sum_{i}\left[G_{i}\left(\phi\left(l_{i}\right)-G_{i}\left(\phi\left(u_{i}\right)\right)\right]\right.
\end{aligned}
$$

where

$G_{i}(\phi(x))=\int_{-e \phi(x)}^{-e \phi_{0}} d E\left[\frac{2}{m_{e}}(E+e \phi(x))\right]^{1 / 2} \tilde{f}_{e}^{i}(E, t)$

and the superscript $i$ indicates that $\tilde{f}_{e}^{i}(E)$ is the energy distribution in the $i$ th spatial interval. As an example, let us calculate $\dot{P}_{+}(x)$ in spatial interval 1, i.e. for $x_{0}<x<x_{1}$. From Eq. (33) we obtain

$$
\begin{aligned}
& \dot{P}_{+}(x)=\left[G_{1}(\phi(x))-G_{1}\left(\phi_{1}\right)\right]+\left[G_{2}\left(\phi_{1}\right)-G_{2}\left(\phi_{2}\right)\right] \\
& \quad+\left[G_{3}\left(\phi_{2}\right)-G_{3}\left(\phi_{3}\right)\right]+\ldots
\end{aligned}
$$

When $G_{i}(\phi)$ is evaluated at a potential energy peak, for example at $\phi=\phi_{1}$, the integral for $G_{i}(\phi)$ involves $f_{e}(E)$ for reflecting particles only, i.e. $f_{0 e}^{+}(E)$. Since $f_{0 e}^{+}(E)$ is the same function of $E$ in all spatial regions, $G_{i}(\phi)$ evaluated at a potential energy peak does not depend on the spatial interval $i$. Thus it follows that in the sum above, the last term in each bracket exactly cancels the first term in the succeeding bracket. The only surviving terms are the first term of the first bracket and the last term of the last bracket. We obtain for $x_{0}<x<x_{1}$

$$
\begin{aligned}
\dot{P}_{+}(x) & =G_{1}(\phi(x))-G_{\infty}\left(\phi_{+\infty}\right) \\
= & G_{1}(\phi(x))-\int_{0}^{-e \phi_{0}} d E\left(\frac{2 E}{m_{e}}\right)^{1 / 2} \tilde{f}_{0 e}^{+}(E) .
\end{aligned}
$$


To calculate $S_{+}(x)$ from (19) we need, in addition to $\dot{P}_{+}(x)$, $e \phi \tilde{n}_{e}$ which can be written

$$
\begin{gathered}
e \phi \tilde{n}_{e}=\int_{-e \phi(x)}^{-e \phi_{0}} d E\left[\frac{2}{m_{e}}(E+e \phi(x))\right]^{1 / 2} \\
\tilde{f}_{e}^{1}(E)\left[1-\frac{E}{E+e \phi(x)}\right] .
\end{gathered}
$$

Using Eq. (34) this becomes

$$
\begin{aligned}
e \phi \tilde{n}_{e} & =G_{1}(\phi(x)) \\
& -\left(2 / m_{e}\right)^{1 / 2} \int_{-e \phi(x)}^{-e \phi_{0}} d E \frac{E \tilde{f}_{e}^{1}(E)}{[E+e \phi(x)]^{1 / 2}} .
\end{aligned}
$$

Using Eqs. (36) and (38) in Eq. (19), we obtain

$$
\begin{gathered}
S_{+}(x)=\left(2 / m_{e}\right)^{1 / 2} \int_{-e \phi(x)}^{-e \phi_{0}} d E \frac{E \tilde{f}_{e}^{1}(E)}{[E+e \phi(x)]^{1 / 2}} \\
-\left(2 / m_{e}\right)^{1 / 2} \int_{0}^{-e \phi_{0}} d E E^{1 / 2} \tilde{f}_{0 e}^{+}(E) .
\end{gathered}
$$

Following a similar procedure for $x<x_{0}$ we obtain

$$
\begin{aligned}
& S_{-}(x)=-\left(2 / m_{e}\right)^{1 / 2} \int_{-e \phi(x)}^{-e \phi_{0}} d E \frac{E \tilde{f}_{e}^{-1}(E)}{[E+e \phi(x)]^{1 / 2}} \\
& +\left(2 / m_{e}\right)^{1 / 2} \int_{0}^{-e \phi_{0}} d E E^{1 / 2} \tilde{f}_{0 e}^{-}(E)
\end{aligned}
$$

where $\tilde{f}_{e}^{-1}(E)$ in the first integral is the distribution function for the first spatial region on the left hand side of the main peak and $\tilde{f}_{0 e}^{-}(E)$ is the distribution function for untrapped electron for $x<x_{0}$.

We now evaluate $S_{+}(x)$ at the location of the central peak, i.e. at $x=x_{0}$ where $\phi=\phi_{0}$.

$S_{+}\left(x_{0}\right)=-\left(2 / m_{e}\right)^{1 / 2} \int_{0}^{-e \phi_{0}} d E E^{1 / 2} \tilde{f}_{0 e}^{+}(E)$.

If in the absence of a wavepacket the distribution function is $f_{0 e}(v)$ then for $|v|<s_{0}=\left(-2 e \phi_{0} / m_{e}\right)^{1 / 2}$

$f_{0 e}^{+}(E)=f_{0 e}(u-|v|) \quad, \quad f_{0 e}^{-}(E)=f_{0 e}(u+|v|)$.

Using this in Eq. (30) we obtain

$\tilde{f}_{0 e}^{+}=f_{0 e}(u-|v|)-f_{0 e}(v) \approx-2 f_{0 e}^{\prime}(u) v$.

Substituting this into Eq. (41) we find

$S_{+}\left(x_{0}\right)=2 m_{e} f_{0 e}^{\prime}(u) \int_{0}^{s_{0}} v^{3} d v=\frac{\omega_{p e}^{2}}{2 \pi} f_{0 e}^{\prime}(u) \phi_{0}^{2}$.

When $f_{0 e}^{\prime}(u)>0$ we obtain the physically plausible result that $S_{+}\left(x_{0}\right)>0$.

The calculation of $S_{-}\left(x_{0}\right)$ is completely analogous to $S_{+}\left(x_{0}\right)$, and we find that $S_{-}\left(x_{0}\right)=S_{+}\left(x_{0}\right)$. The total rate of electron momentum loss from the electrons and transferred to the wave packet is

$$
\begin{aligned}
\dot{P} & =S_{+}\left(x_{0}\right)+S_{-}\left(x_{0}\right)=\dot{P}_{+}\left(x_{0}\right)+\dot{P}_{-}\left(x_{0}\right) \\
& =\omega_{p e^{2} \pi^{-1}}^{2} f_{0 e}^{\prime}(u) \phi_{0}^{2} .
\end{aligned}
$$

We can also evaluate $S_{+}(x)$ at the point $x=x_{s}$ where the potential $\phi(x)$ has its first zero on the right hand side of $x_{0}$. From Eq. (39)

$S_{+}\left(x_{s}\right)=\int_{0}^{-e \phi_{0}} d E E^{1 / 2}\left[f_{e}^{1}(E)-f_{0 e}^{1}(E)\right]$.

The tilde disappears from the distribution functions since the $f_{0 e}$ 's in Eq. (30) cancel. To evaluate this expression, one needs to know $f_{e}(E)$ for region 1 . One can relate $f_{e}^{1}(E)$ to $f_{0 e}^{+}(E)$, the distribution function for untrapped particles valid for $x_{0}<x<\infty$. An electron of energy $E$ at time $t$ was trapped (captured) at an earlier time $t_{c}$ when its energy was $E_{c}$. Since phase space density is a constant along particle orbits, one can set $f_{e}^{1}(E, t)=f_{0 e}^{+}\left(E_{c}\right)$. Using this result in Eq. (46), the last factor in the integrand becomes

$\left[f_{0 e}^{+}\left(E_{c}\right)-f_{0 e}^{+}(E)\right]$.

It seems apparant as we will prove, that for a growing wave packet, the energy of an electron decreases after it becomes trapped, i.e. $E=m_{e} v^{2} / 2-e \phi<E_{c}$. This result is obvious for the special case of a square well. For a square wave $\partial \phi(x) / \partial x=0$ except at the well edge where it is infinite. The electron velocity is constant except that it changes sign when it is reflected at the well edge. Therefore the kinetic energy is constant and the electron energy $E$ decreases as the well depth increases, i.e. as $-e \phi$ assumes a larger negative value. Indeed it is easy to show that the total energy of an electron $E$ satisfies the following equation

$\frac{d E}{d t}=-e \frac{\partial \phi}{\partial t}$

integrating this equation (48) over time from the time $t_{c}$ to alater time $t>t_{c}$ leads to:

$E(t)-E_{c}=-e \int_{t_{c}}^{t} d \tau \frac{\partial \phi(x(\tau), \tau)}{\partial \tau}$

if we now express that the potential is growing and that $\partial \phi / \partial t=\gamma \phi$, with $\gamma>0$, then the expression for the energy difference Eq. (49) becomes

$E(t)-E_{c}=\int_{t_{c}}^{t} d \tau[-e \gamma \phi(x(\tau), \tau)]$

let us look at times $t=t_{c}+\delta t$ with $\omega_{b} \delta t \ll 1$, where $\omega_{b}$ is the electron bounce frequency. Then we have

$E(t)-E_{c} \approx \gamma \delta t E_{c}$

but since $E_{c}<0$ for trapped electrons, we therefore conclude that $E<E_{c}$ for a general wave form.

Referring to Eq. (42), we see that $f_{0 e}^{+}(v)$ is a decreasing function of $|v|$ and therefore of $E=m_{e} v^{2} / 2$ for $E<-e \phi_{0}$. This means that $f_{0 e}^{+}\left(E_{c}\right) \leq f_{0 e}(E)$ and that the factor Eq. (47) is negative and therefore that $S_{+}\left(x_{s}\right)<0$. We have previously shown that $S_{+}\left(x_{0}\right)>0$. It follows that $S_{+}(x)$ must vanish for $x=x_{+}$where $x_{0}<x_{+}<x_{s}$. A similar argument shows that $S_{-}(x)$ vanishes for $x=x_{-}$where $x_{-}$lies between $x_{0}$ and the first zero of the potential to the left of $x_{0}$. Demonstrating the existence of these zeros of $S_{-}(x)$ and $S_{+}(x)$ justifies Eq. (24). 


\section{Summary}

Equation (24) can be used to determine the growth of $\phi^{2}$ in the region $x_{-}<x<x_{+}$. We approximate

$\int_{x_{-}}^{x_{+}} d x \phi(x)^{2} \approx(\pi / k) \phi_{0}^{2}$

and we use Eqs. (3) and (45) to write

$2 k c_{s} \lambda_{D}^{2}\left[\dot{P}_{-}\left(x_{0}\right)+\dot{P}_{+}\left(x_{0}\right)\right]=2 \gamma_{\ell} \phi_{0}^{2}$.

Dispersion will cause a loss of momentum from the region of the main peak which we can estimate from $J$ given by Eq. (18) and using $\partial / \partial x \approx k$. Thus

$2 k c_{s} \lambda_{D}^{2}\left[J\left(x_{-}\right)-J\left(x_{+}\right)\right] \approx 2 \gamma_{d} \phi_{0}^{2}$.

Using all these approximations in Eq. (24) and setting $\partial \phi / \partial t=\gamma \phi$, we obtain for the growth rate of the main peak of the wave packet

$\gamma \approx \gamma_{\ell}-\gamma_{d}$

This is the same result that was argued in the Introduction and implies that the main peak of a wave packet will grow in amplitude if the linear growth rate exceeds the dispersion rate.

In the 3-D case, dispersion causes the wave packet to broaden in three dimensions, i.e. both parallel and perpendicular to the electron current. Although we have not done a detailed calculation, preliminary studies of the three dimensional case with a magnetic field along the direction of current flow show that in the direction of the field lines the 1-D result holds, i.e. the electron momentum is deposited in a spatial region of the width of the main peak. In the perpendicular direction it is obvious that all of the momentum lost by those reflected electrons lying in the cross section of the main peak will be deposited in the main peak since the electrons are constrained to move along the field lines. Perpendicular dispersion does not cause a reduction in the momentum input to the main peak. Thus the main peak will grow if the linear growth rate exceeds the parallel dispersion rate. In the directions perpendicular to the electron current, the dispersive spreading of the potential will increase the total cross section of the wave packet but this will be balanced by an increasing number of reflecting electrons.

\section{Conclusions}

In conclusion, a proof based on a fundamental physical principle has been put forward to explain the evolution of ion acoustic wave packets into the nonlinear phase. It is often the case in ion acoustic turbulence to take into account the ion nonlinearity, but it has been shown in this paper that the electron nonlinearities can be equally important, and can provide the mechanisms necessary to sustain large amplitude ion fluctuations. Two fundamental arguments have been put forward to understand the development and evolution of large amplitude ion fluctuations. The first argument relies on the fact that isolated structures will likely evolve to larger amplitudes than the amplitudes of packed ion structures, while the second argument suggests that early on in the evolution of the ion structures, the electron nonlinearities play a more significant role than the ion ones. Conventional wisdom has it that the ion nonlinearity would allow for the development of KdV like turbulence with solitary structures. The structures that evolve from a KdV like description of a two-species drifting plasma are purely compressive as opposed to rarefactive. Moreover, a sign difference in the nonlinearity would lead to wave-like Airy function solutions to the nonlinear KdV equation. The solutions to the $\mathrm{KdV}$ equation are very sensitive to the initial conditions too. Conventional wisdom also suggests that growth is associated with momnetum input. However we have shown that only the main peak grows and that the secondary peaks do not. Clearly, this is due to the fact that there is a net momentum input by the electrons to the main peak, which then feeds the secondary peaks through dispersion. The secondary peaks do not compete, as we have argued, for the nonlinear electron momentum; They live in the shadow of the dominant peak indeed, so they tend to decay.

We have argued that, and quantified our arguments in this paper, the electron nonlinearity can be a fundamental player in the development of isolated, selfconsistent nonlinear structures. The reflection and trapping of electrons early on in the development of ion acoustic structures plays a fundamental role in determining the existence of BGK like equilibria.

Ion acoustic turbulence is believed to play a significant role in auroral physics. One of the fundamental issues in turbulence is to understand the seeding mechanisms for large amplitude fluctuations. The present study suggests that given the linear instability conditions an emitted wave packet can evolve into a large amplitude structure by tapping the electron momentum. Moreover, an argument based on the probability distribution of fluctuations favors the evolution of turbulence towards a state of large amplitude isolated structures.

The proposed model relies heavily on momentum conservation. A balance between linear momentum dispersion and nonlinear momentum input from electrons is shown to occur when the linear plasma instability growth rate is larger than the dispersion rate. Moreover, it is shown that a net momentum input goes directly to the peak of a linearly excited ion acoustic wave packet.

Finally, a complete model would have to be three dimensional with the inclusion of a background magnetic field. A study that involves multiple structures would have to be put forward in order to extrapolate the results of the one dimensional model presented in this paper.

Acknowledgements. The author would like to thank J.-P. St-Maurice for a number of discussions on the subject of intermittency.

Topical Editor T. Pulkkinen thanks two referees for their help in evaluating this paper. 


\section{References}

Barnes, C., Hudson, M. K., and Lotko, W.: Weak double layers in ion acoustic turbulence, Phys. Fluids, 28, 1055-1062, 1985.

Berman, R. H, Dupree, T. H., and Tetrault, D. J: Growth of nonlinear intermittent fluctuations in linearly stable and unstable simulation plasma, Phys. of Fluids , 29, 2860-2870, 1986.

Berman, R. H, Dupree, T. H, Tetrault, D. J, and Boutros-Ghali, T.: Computer simulation of nonlinear ion-electron instability, Phys. Rev. Lett., 48, 1249-1252, 1982.

Bernstein, I. B., Greene, J. M., and Kruskal, M. D.: Exact nonlinear plasma oscillations, Phys. Rev., 108, 546-550, 1957.

Davidson, R. C., Galeev, A. A., and Sudan, R. N. (Eds.): Kinetic waves and instabilities in a uniform plasma, Vol. 1, NorthHolland Pub. Co., New York, 519-587, 1983.

Denavit, J. and Sudan, R. N.: Effect of trapped particles on the nonlinear evolution of a wave packet, in: Effect of trapped particles on the nonlinear evolution of a wave packet, Phys. Rev. Lett., 28, 404-407, 1972.

Dupree, T. H.: Growth of phase space holes near linear instability, Phys. of Fluids, 29, 1813-1819, 1986.

Drummond, W. E.: Growth of phase space holes near linear instability, in: Quasi-Linear theory of plasma turbulence, International Atomic Energy Agency, International Centre for Theoretical Physics, Trieste, October 5-31, 1964, Seminar on plasma physics, Vienna, the Agency, 527-543, 1965.

Frisch, U.: Turbulence, Cambridge University Press, 1995.

Hamza, A. M.: Development of intermittent, spatially localized ion fluctuations, Ph.D Thesis, M.I.T., 1988.

Hamza, A. H.: Nonlinear-electron-response impact on the evolution of ion-acoustic wave packets in a magnetized plasma, Physical Review E, 48, 2055-2066, 1993.

Kadomtsev, B. B.: Plasma turbulence: general topics, Plasma Physics, International Atomic Energy Agency, Lectures presented at the seminar on Plasma Physics, organized and held at the International Centre for Theoretical Physics, Trieste, October 5-31, 1964, Seminar on plasma physics, Vienna, the Agency, 1965.
Karpman, V. I.: The effects of the interaction between Ion-Sound solitons and resonance particles in a plasma, in: The effects of the interaction between Ion-Sound solitons and resonance particles in a plasma, Sov. Phys. JETP, 40, 695, 1979.

Koeskinen, H. E. J., Holback, B., Bostrom, R., and Gustafson, G.: Viking Observations of solitary waves and weak double layers on auroral field lines, SPI conference proceedings and reprint series, Physics of Space Plasmas (1987), Proceedings of the 1987 Cambridge workshop in geoplasma physics Ionosphere-Magnetosphere-Solar Wind coupling processes, edited by: Chang, T., Crew, G. B., and Jasperse, J. R., 147-157, 1988.

Manheimer, W.: Strong Turbulence Theory of Nonlinear Stabilization and Harmonic Generation , Phys. of Fluids, 14, 579-590, 1971.

Mozer, F. S., Temerin, M., Cerny, K., and Lotko, W.: Observations of Double Layers and Solitary Waves in the Auroral Plasma , Phys. Rev. Lett., 48, 1175-1179, 1982.

Pottelette, R., Treumann, R. A., and Georgescu, E.: Crossing a narrow-in-altitude turbulent auroral acceleration region, Nonlin. Processes Geophys., 11, 197-204, 2004,

SRef-ID: 1607-7946/npg/2004-11-197.

Tetrault, D. J.: Growing ion holes as the cause of auroral double layers, J. Geophys. Res. Lett., 15, 164-168, 1988.

Sato, T. and Okuda, H.: Numerical simulations on ion accoustic double. layers, J. Geophys. Res., 86, 3367-3368, 1981.

Sato, T. and Okuda, H.: Ion-Acoustic Double Layers, Phys. Rev. Letter, 44, 740-743, 1979.

Taniuti, T.: Ion-Acoustic Solitary Waves with effects of Resonant particles, Supp. of the Prog. of Theor. Phys., 55, 191-212, 1974. 